\title{
Fluoxetine and Oculomotor Activity during Sleep in Depressed Patients
}

Roseanne Armitage, Ph.D., Madhukar Trivedi, M.D., and A. John Rush, M.D.

The effects of fluoxetine on automated measures of electrooculographic (EOG) and electromyographic (EMG) activity were evaluated in 41 patients with nonpsychotic, major depressive disorder. Sleep EEG evaluations were conducted at baseline, while patients were symptomatic and unmedicated, and following four to five weeks of treatment with fluoxetine $(20 \mathrm{mg})$. The number of eye movements $(>75 \mu \mathrm{V})$ and the amplitude of EOG and $E M G$ activity increased significantly on treatment in
REM, stages 1, 2, and slow-wave sleep. All patients showed EOG and EMG abnormalities in at least one stage of sleep. Thirty-four percent of patients showed increased EOG and EMG activity on treatment in every sleep stage. It is suggested that fluoxetine-induced oculomotor abnormalities are likely to be the result of increased availability of serotonin and secondary dopaminergic effects. [Neuropsychopharmacology 12:159-165, 1995]
KEY WORDS: Sleep; Depression; Antidepressants; Fluoxetine.

Fluoxetine is a 5-hydroxytryptamine (5-HT) reuptake inhibitor that has equivalent antidepressant efficacy to tricyclic medications (Depression Guidelines Panel 1993). Several studies have shown that fluoxetine disrupts sleep, resulting in decreased sleep efficiency, increased light (stage 1) sleep and nocturnal wakefulness, and is associated with an increase in complaints of insomnia (Cooper 1988; Hendrickse et al. 1994; Nicholson and Pascoe 1988). Moreover, fluoxetine has been shown to produce unusual eye movements throughout non-rapid eye movement (NREM) sleep (Keck et al. 1990, 1991). In a retrospective study of 40 depressed and one obsessive-compulsive patient, Schenck et al. (1992) reported fluoxetine-induced eye movements in NREM sleep in about $50 \%$ of the sample. In a preliminary report, Dorsey et al. (1992) also found increased

From the University of Texas Southwestern Medical Center at Dallas, TX.

Address correspondence to: Roseanne Armitage, Ph.D., The University of Texas Southwestern Medical Center, 5323 Harry Hines Boulevard, Dallas, TX 75235-9070.

Received July 13, 1993; revised October 4, 1994; accepted October 10, 1994 myoclonic and eye movement activity during NREM sleep in about $60 \%$ of patients treated with fluoxetine. None of these studies, however, quantified the number or amplitude of fluoxetine-induced oculomotor abnormalities.

The present study was conducted to evaluate the impact of fluoxetine on automated measures of electromyographic (EMG) and electrooculographic (EOG) activity during sleep in depressed outpatients.

\section{METHODS}

\section{Subjects}

The study sample was chosen from a pool of men and women, 18 to 65 years of age, who met DSM-III-R criteria for nonpsychotic, major depression, but who were otherwise medically healthy. Patients who were currently engaged in shiftwork and those with independent sleep disorders (e.g., narcolepsy, apnea, bruxism, myoclonus, etc.) established by history or polysomnogram $(n=4)$ were excluded from study. Other current Axis I disorders, current general medical conditions, or psychoactive substance use within the previous 12 months also excluded patients from study. In addition, women were excluded if they were pregnant 
or lactating or were not using an adequate method of contraception. All patients provided written informed consent to participate in the study.

Diagnoses were made based on the Structured Clinical Interview for DSM-III-R (Spitzer et al. 1986). The 17-item Hamilton Rating Scale (HRS-D) (Hamilton 1960) was also used to assess symptom severity, and a detailed personal and family history was obtained at the time of clinical interview. Forty-one outpatients with major depression ( 17 men, 24 women), averaging $42.9 \pm$ 9.9 years of age, participated in the study. All patients were medication-free for a minimum of 2 weeks at the time of study. None had been treated previously with selective serotonin reuptake inhibitors (SSRIs). Patients were part of a larger, ongoing study $(n=60)$ on the effects of fluoxetine on sleep. Data analyses are not yet available for the remaining 19 patients. A detailed description of the clinical and demographic characteristics of the sample is provided in Table 1.

\section{Procedure}

Each subject maintained individualized, regular bedand rise times for 5 days prior to sleep study, as assessed by home diary. The subjects spent two consecutive, baseline nights in the University of Texas Southwestern Medical Center Sleep Study Unit. Patients returned to the laboratory for two additional nights after 4 to 5 weeks of daily treatment of fluoxetine ( $20 \mathrm{mg}$ q.d. A.M.) No additional medications or psychotherapy were used. To rule out adaptation effects, data from the second

Table 1. Sample Features of Major Depressive Disorder (MDD) ${ }^{a}$

17-Item HRS-D

Baseline: $21.2 \pm 3.6$ (range: 16 to 29 )

On treatment: $9.8 \pm 6.2$ (range: 0 to 32 )

Patients with $50 \%$ decrease in HRS-D from baseline $(n=24)$

Course of illness

First episode $(n=16)$

Recurrent complete $(n=15)$

Recurrent incomplete $(n=10)$

Age at onset $(26.1 \pm 11.3$; range: 8 to 49 years)

Length of current episode $(n=38 ; 42.4 \pm 54$; (range:

3 to 27 months)

Depression subtype

RDC endogenous $(n=20)$

RDC probably endogenous $(n=15)$

RDC nonendogenous $(n=5)$

DSM-III-R melancholic $(n=16)$

DSM-III-R nonmelancholic $(n=24)$

Family history of MDD

Positive $(n=20)$

Negative $(n=14)$

Unknown $(n=6)$

a Total sample: $n=41 ; 17$ males; 24 females; $n<41$, missing data. night of study at each recording occasion are reported here.

Electroencephalographic data were collected from left (C3) and right (C4) central electrodes with a common reference (right and left ear lobes passed through a $10-\mathrm{k} \Omega$ resistor). Monopolar, left and right EOG, and bipolar chin-cheek EMG data were also recorded. A full electrode montage, used on the first night in the sleep laboratory, included leg, chest, and abdomen leads and a nasal-oral thermistor to rule out independent sleep disorders. All electrophysiological signals were transduced by GRASS ${ }^{\mathrm{TM}}$ P-511 AC amplifiers and displayed on a paperless polygraph system. An amplifier sensitivity of 5 was used for EEG $(50-\mu \mathrm{V}, 0.5$-sec duration calibration), with half-amp low- and high-bandpass filters set at 0.3 and $30 \mathrm{~Hz}$, respectively. $A 60-\mathrm{Hz}$ notch filter attenuated electrical noise. The EMG amplifiers were set at a sensitivity of 1 , with half-amp high filters at 30 and low filters set at $0.1 \mathrm{~Hz}$. Electrooculographic amplifiers were set at a sensitivity of 5, with half-amp high filters set at 30 and low filters set at $0.1 \mathrm{~Hz}$. Interelectrode impedances were maintained below $2 \mathrm{k} \Omega$. Amplifiers were calibrated before and after each night's sleep. Data were digitized on-line through a 16-bit MICROSTAR ${ }^{\mathrm{TM}}$ analogue-to-digital (A/D) board (EEG sampled at $250 \mathrm{~Hz}$ and EOG and EMG sampled at 62.5 $\mathrm{Hz}$ ) and stored on optical disk for off-line analysis and stage scoring (Armitage et al. 1994).

Automated analyses were conducted on EOG and EMG activity in 30-sec epochs. Similar EOG and EMG algorithms have been described elsewhere (Hoffmann et al. 1979). The algorithm computes the number of eye movements in each EOG channel larger or equal to 75 $\mu \mathrm{V}$ [EOG count (EOGC)]. An eye movement was defined as any positive or negative voltage excursions that hit threshold beyond baseline $(0 \mathrm{~V})$. The EOG threshold of $75 \mu \mathrm{V}$ was determined through repeated adjustments in sensitivity, until the detected events matched those that would be considered eye movements based on visual inspection. No duration criterion was applied to eye movement count, nor were simultaneous eye movements required in the two EOG channels. The EOG counts were then averaged for the right and left channels, producing an average EOG count per 30 -sec epoch. The EOG amplitude was computed as the total cumulative voltage of the EOG signal [EOG amplitude (EOGA)], with no minimum threshold. The EOG amplitude was then averaged for the right and left channels for each 30-sec epoch. The total cumulative voltage of the EMG signal defined EMG amplitude [EMG amplitude (EMGA)] in each 30-sec epoch, with no minimum threshold.

Sleep EEG records were scored from C3 according to standard Rechtschaffen and Kales (1968) criteria by technicians trained at better than $90 \%$ agreement on an epoch-by-epoch basis. Measures were combined for 
stages 3 and 4 sleep (SW), as few subjects showed stage 4 sleep either at baseline or on treatment. All statistical analyses were conducted using $S A S^{\mathrm{TM}}$ routines. Data were inspected visually to remove epochs with movement artifact or episodes of wakefulness $(\geqslant 16 \mathrm{sec})$. The stage scores [rapid eye movement (REM), stages 1, 2, and $S W]$ were then used to categorize EOG and EMG measures by sleep stage.

Statistical Procedures. Means and standard deviations were computed for EOGC, EOCA, and EMGA in each sleep stage, and data were coded by gender and treatment level (baseline versus on-treatment). Separate split-plot analyses of variance (ANOVAs) were computed on EOGC, EOGA, and EMGA, with gender as the between-group variable. Sleep stage (REM, stages 1,2, and SW) and treatment level were used as repeated measures.

\section{RESULTS}

Every subject showed increased activity from baseline in at least one measure of oculomotor activity. A visual representation of the fluoxetine-induced EOG abnormalities is shown in Figure 1. Note that both fast and slow eye movements are present in this epoch of stage
2 sleep and that eye movements are not highly correlated with EEG changes. In terms of computerquantified measures, 14 of the 41 patients (34\%) showed increased EOG and EMG activity on fluoxetine in all sleep stages. Increased EMG amplitude was found in $33 / 41$ subjects $(80 \%)$. Numerically increased EOG count was apparent in $29 / 41$ patients $(71 \%)$, whereas increased EOG amplitude in all stages was evident in $30 / 41$ patients $(75 \%)$. Significant effects are described below.

\section{EOG Count}

Significant treatment $(F=21.9, \mathrm{df}=1,39, p<.0001)$, sleep stage $(F=40.3, \mathrm{df}=4,156, p<.0001)$, and treatment by stage interactions $(F=2.6$; df $=4,156, p<$ $.04)$ were obtained for the EOGC. No significant gender main effects or interactions were found. Figure 2 illustrates the effects of fluoxetine on EOGC in each sleep stage. The EOGC was increased in all stages. Stage 1 and REM sleep were associated with the largest increases in EOGC from baseline to treatment, accounting for the sleep stage by treatment interaction. Univariate ANOVAs indicated significant treatment effects for all sleep stages (range $F=4.79$ to $17.77, \mathrm{df}=1,39, p=$ .04 to .0001$)$.

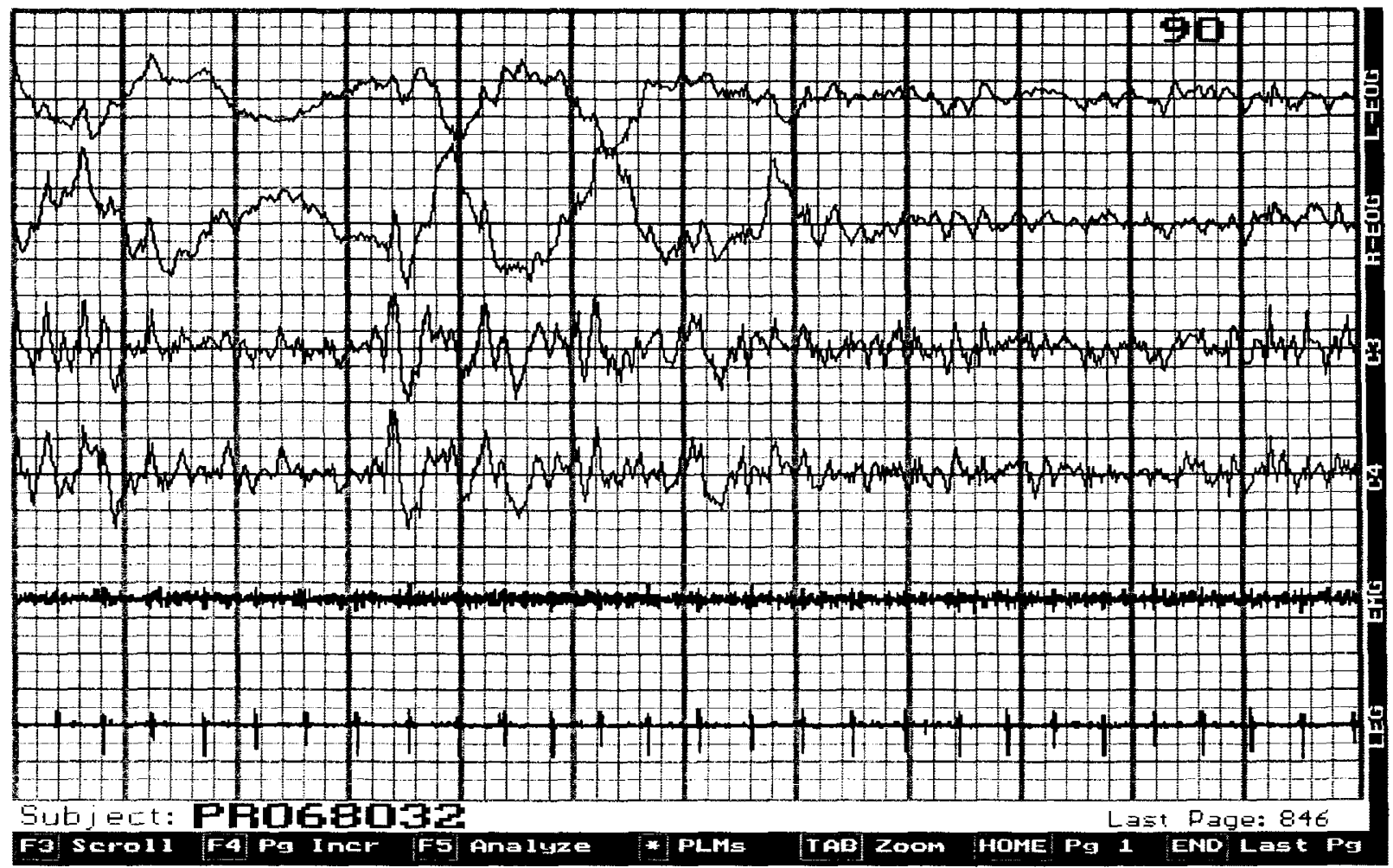

Figure 1. Fluoxetine-induced movements in a 30-sec epoch of stage 2 sleep from a representative depressed patient. Polygraphic channels are left EOG, right EOG, C3, C4, EMG, and bipolar leg leads. Each block of the polygraphic grid is of 0.5 -sec duration and $25-\mu \mathrm{V}$ amplitude. 


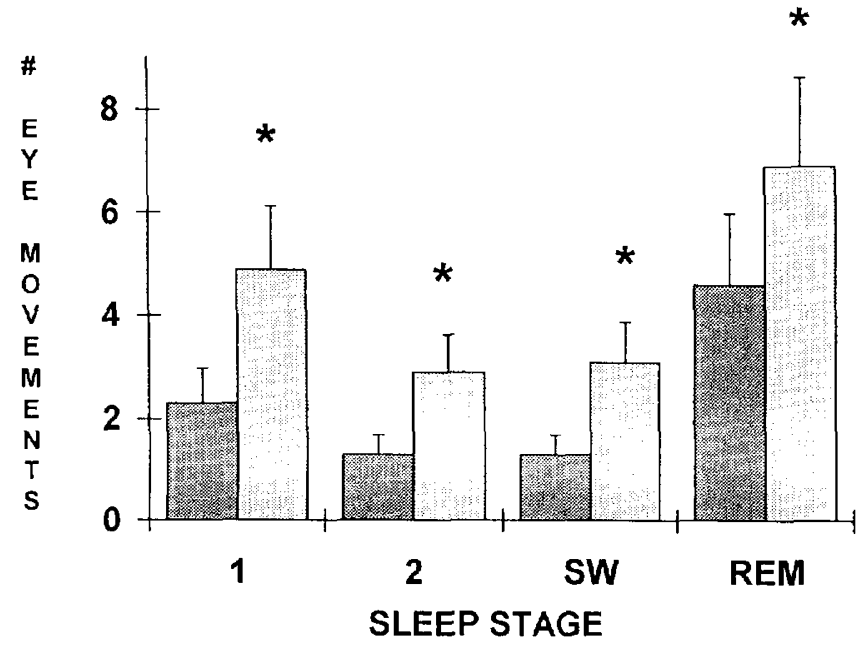

Figure 2. EOG count: average number of eye movements (EMS) $>75 \mu \mathrm{V}$ per 30-sec epoch in all sleep stages at baseline (网) and following fluoxetine treatment ([1). Asterisks denote significant treatment main effects $(p<.05)$. Error bars denote standard deviations.

\section{EOG Amplitude}

Significant main effects for treatment $(F=25.04$; $\mathrm{df}=$ $1,39 ; p=.0001)$ and sleep stage $(F=30.07, \mathrm{df}=4,156$, $p=.0001$ ) were found for EOGA. Treatment by gender and treatment by sleep-stage interactions were also obtained $(F=4.74, \mathrm{df}=1,39, p=.04 ; F=4.16, \mathrm{df}=$ $4,156, p=.003$, respectively), along with a trend toward a gender by sleep-stage interaction $(F=2.29, \mathrm{df}=$ $4,156, p=.063$ ). These effects are illustrated in Figure 3. As in the analysis of the number of eye movements, fluoxetine-induced increases in EOGA were largest in stages 1 and REM sleep. Univariate ANOVAs of EOGA revealed significant treatment main effects for stages 1 and REM sleep $(F=35.94, \mathrm{df}=1,39 ; p=.0001 ; F=$ 13.33, $\mathrm{df}=1,39, p=.0008$ ). In SW EOGA showed a significant treatment by gender interaction $(F=4.56$, $\mathrm{df}=1,39, p=.04)$. Males showed smaller increases in EOGA in SW sleep than did females. In stage 2 sleep EOGA showed a significant treatment effect $(F=26.71$, $\mathrm{df}=1,39, p=.0001)$ and a trend toward a treatment by gender interaction $(F=3.53, \mathrm{df}=1,39, p=.07)$.

\section{EMG Amplitude}

Significant treatment and sleep stage main effects were obtained from ANOVA of EMGA $(F=30.33, \mathrm{df}=1,39$, $p=.0001 ; F=79.91, \mathrm{df}=4,156, p=.0001$, respectively). A trend toward a treatment by sleep-stage interaction was also evident $(F=2.34, \mathrm{df}=4,156, p=$ .06). Means indicated an increase in EMGA in all sleep stages from baseline to treatment as seen in Figure 4. Univariate analyses confirmed significant treatment
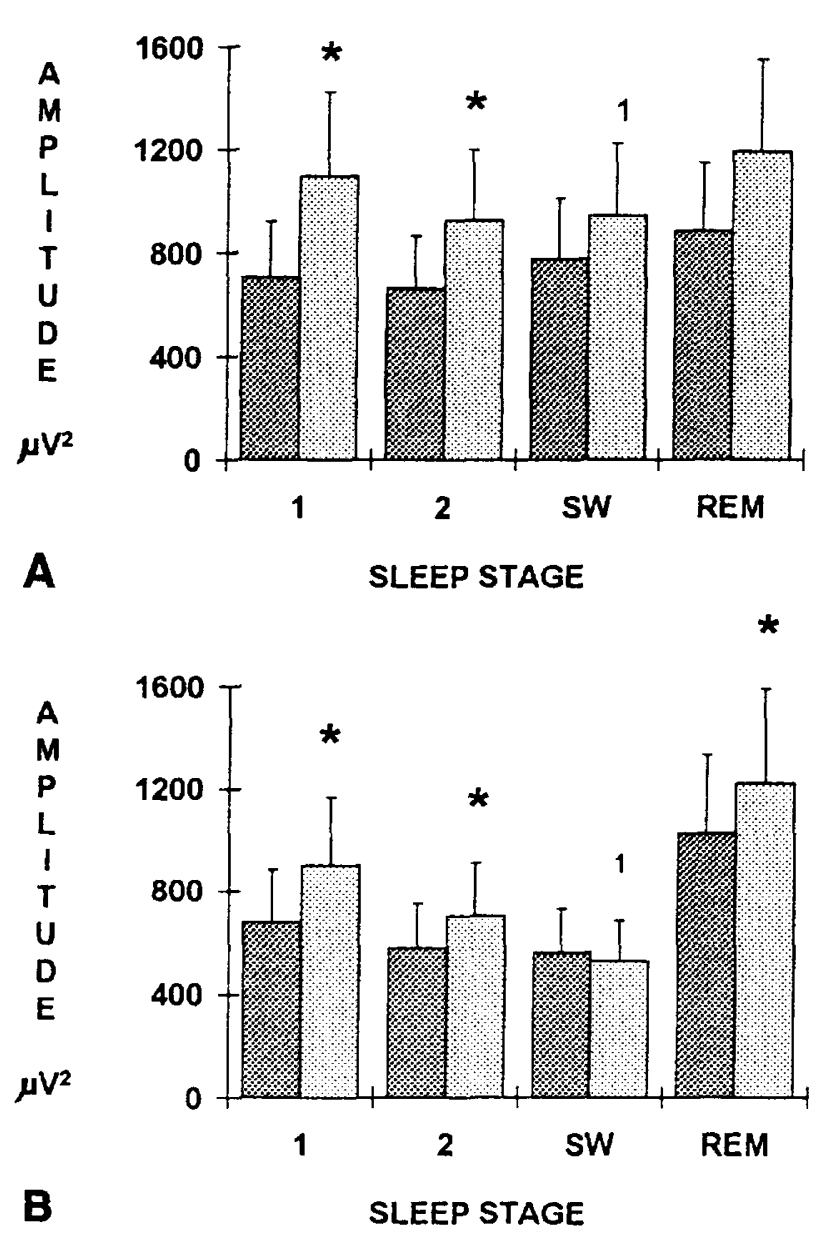

Figure 3. EOG amplitude: $\mathbf{A}$, males $(n=17)$; $\mathbf{B}$, females $(n=$ 24). Average eye movement amplitude measured in $\mu \mathrm{V}^{2}$ per 30-sec epoch in all sleep stages at baseline (ख) and following

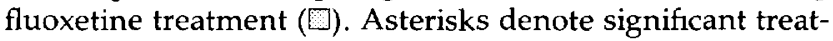
ment main effects. Superscripted 1 over columns denotes significant gender by treatment interaction $(p<.05)$. Error bars denote standard deviations.

effects for all sleep stages (range $F=4.47$ to 41.16 , $\mathrm{df}=$ $1,39, p=.04$ to .0001 ), with largest increases in EMGA from baseline to treatment in stages 1 and 2 sleep. No gender differences were found.

\section{DISCUSSION}

To summarize, the number of eye movements, the amplitude of eye movements, and the amplitude of EMG activity increased significantly following 4 to 5 weeks of fluoxetine treatment. These effects were evident for all oculomotor measures in every sleep stage, except for EOGA in slow-wave sleep. However, EOG amplitude did show treatment differences along gender lines. Means indicated that fluoxetine-induced increases in 


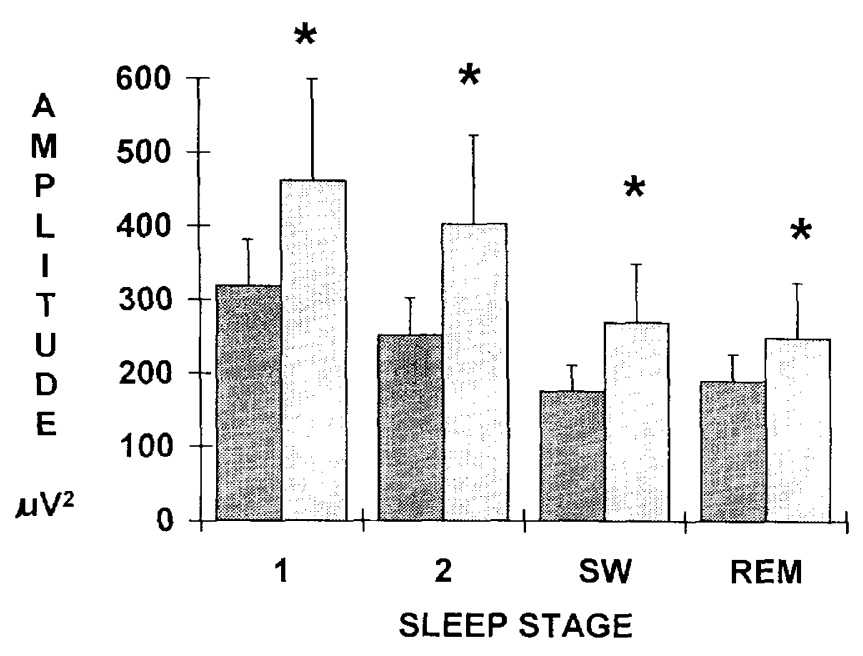

Figure 4. EMG amplitude: average EMG amplitude mea-

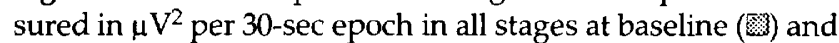
following fluoxetine treatment (U). Asterisks denote significant treatment main effects $(p<.05)$. Error bars denote standard deviations.

EOGA were larger among women. No other gender differences were found.

Fluoxetine-induced abnormalities were evident in at least one EOG or EMG measure in all patients. In fact, fluoxetine-induced increases in every measure, in all sleep stages, were apparent in over $30 \%$ of the sample (14/41 patients). There was, however, a wide range of variability about the increases, indicating that some patients were affected more dramatically than others. It may aid our understanding of the mechanisms underlying fluoxetine-induced activation to identify the clinical and biological characteristics of patients who do not show oculomotor increases on treatment or those who do with only minor effects.

Our findings are in keeping with recent reports of fluoxetine-induced eye movements in NREM sleep based on visual scoring of EOG activity (Dorsey et al. 1992; Keck et al. 1990, 1991; Schenck et al. 1992). Moreover, we found an increase in the number and the amplitude of eye movements in both REM and NREM sleep. In contrast to our reports, previous findings have indicated that these effects are more pronounced in stage 1 sleep (Keck et al. 1990, 1991; Schenck et al. 1992). The results of the present study suggest that treatment effects are roughly equivalent in stages 1 and REM sleep. It should be noted, however, that the algorithm for EOGC only detected eye movements in excess of $75 \mu \mathrm{V}$, and as such, may quantify fewer eye movements than are apparent in visual analyses. Thus, our analysis is likely to provide a conservative estimate of EOG events, perhaps accounting for the discrepancy between studies. It is possible that fluoxetine is also likely to increase the number of eye movements below $75 \mu \mathrm{V}$. Using a lower EOG threshold may detect more lower- amplitude fluoxetine-induced eye movements in stage 1 than in other sleep stages. Alternatively, since the amplitude of eye movements is not quantified in visual analysis of REM sleep, previous work may have underestimated the effects of fluoxetine on REM eye movements.

Previous work has suggested that fluoxetineinduced oculomotor abnormalities may be the result of either increased central nervous system (CNS) arousal or a dissociation of EMG and EOG activity from REM sleep (Keck et al. 1990). The latter explanation implies that fluoxetine-induced abnormalities in sleep represent NREM intrusions of REM phasic events. This explanation seems unlikely, since increased EOG and EMG activity were evident in both REM and NREM sleep and may also be present in wakefulness. If REM dissociation was the mechanism underlying fluoxetine-induced eye movements, increased EOGA would not be expected within REM epochs. In addition, the eye movements induced by fluoxetine are not classic, binocularly symmetrical, rapid eye movements. They are better characterized as medium to fast eye movements.

The increase of EMGA found in our patients probably reflects increased myoclonic activity as reported by Schenck et al. (1992). Although we have yet to quantify its incidence, myoclonic activity was noted in about $30 \%$ of our patients. Since patients were excluded if myoclonus was evident at baseline, it seems likely that these effects are due to fluoxetine.

Fluoxetine has also been associated with dyskinesia (Fishbain et al. 1992), akathisia (Lipinski et al. 1989), bruxism (Ellison and Stanziani 1993), and REM behavioral disorder (Schenck et al. 1992). The same mechanism that underlies these motoric side effects probably is responsible for fluoxetine-induced oculomotor abnormalities during sleep. At present, two explanations seem plausible.

The inhibition of serotonin reuptake can produce an excess of available serotonin. Omnipause neurons, responsible for the control of saccadic eye movements (SEMs), are normally inhibited by serotonergic neurons in the brain stem. Schenck et al. (1992) and Baloh et al. (1982) have suggested that the blockade of serotonin uptake may disinhibit omnipause neurons, thus producing SEMs. This explanation may account for abnormal eye movements during sleep, but it does not account for the increase in myoclonic jerks and in muscle activity associated with fluoxetine.

Excessive saccades also have been shown to result from dopamine depletion in wakefulness (Tychsen and Sitaram 1989). Moreover, dyskinesia, akathisia, and extrapyramidal syndrome are more likely to result from a reduction in dopamine (Fishbain et al. 1992) than from increased available serotonin per se. Fluoxetine probably has strong secondary dopaminergic effects by virtue of the inhibitory role serotonin plays on neostriatal 
dopamine function (Seibyl et al. 1989) and the potency of 5-HT reuptake from this compound. Thus, it is likely that fluoxetine-induced oculomotor abnormalities result from primary and secondary serotonergic effects.

Perhaps more important, recent pilot work suggests that fluoxetine-induced eye movements persist 14 months beyond treatment discontinuation (Winkelman et al. 1992). These findings, if replicated, suggest that fluoxetine may have long-term effects on CNS activity. Studies have not been conducted to determine whether other SSRIs have similar effects, although it seems reasonable to assume that oculomotor abnormalities should occur in other SSRIs of equivalent potency.

There are several methodological issues in this study that are noteworthy. In slow-wave sleep EOG are potentially contaminated by delta artifact from the EEG channels, even though the amount of slow-wave activity in this depressed group was less than $5 \%$ of total sleep. However, the correlations between EOG count and amplitude with the amount of delta activity in any given epoch were $r=.18$ and $r=.22$, respectively. In addition, treatment effects were evident in all sleep stages, suggesting that fluoxetine, not EEG contamination, was responsible for the increase in EOG count and amplitude during sleep.

The EMG measure used in this study was also suspect to artifact. Epochs were only excluded from analysis if they contained more than $15 \mathrm{sec}$ of movement or wakefulness, according to stage-scoring criteria (Rechtschaffen and Kales 1968). Thus, the EMG measure may have been contaminated by shorter-duration arousal or movement. Quantifying the number and duration of myoclonic jerks may produce a more reliable measure of fluoxetine effects that is not as suspect to movement artifact.

Finally, the issue of clinical relevance of oculomotor abnormalities requires discussion. Fluoxetine-induced myoclonic activity observed in some patients was intrusive and generally followed by short duration arousal. It is conceivable that increased arousal would be associated with the subjective sense of reduced sleep quality. Nine women $(37.5 \%)$ reported very light, disturbed sleep on fluoxetine, whereas only two males $(11.8 \%)$ reported reduced sleep quality on treatment. Although few gender differences were noted in fluoxetineinduced oculomotor activity, women seemed to be more aware of sleep disruption on treatment. The women who reported reduced sleep quality on fluoxetine also tended to show larger oculomotor increases. The EOG and EMG abnormalities may be a reflection of activating side effects of this antidepressant to which women are more sensitive. Alternatively, these gender differences may simply be a reflection of increased blood levels of fluoxetine or its metabolite norfluoxetine in women of a lower body weight (Hendrickse et al. 1994). A more detailed analysis of the relationship between blood levels, oculomotor activity, sleep effects, and therapeutic response will be conducted following completion of the larger study. In addition, we will study patients after discontinuation to determine the persistence of fluoxetine-induced oculomotor abnormalities.

\section{ACKNOWLEDGMENTS}

We wish to thank the clinical team of the Mental Health Clinical Research Center (A. John Rush, M.D., Director), Kenneth Z. Altshuler, M.D., Chair, Department of Psychiatry, The University of Texas Southwestern Medical Center, for administrative support of this project; the technical staff of the Sleep Study Unit, under the supervision of Darwynn D. Cole, B.S.; and Doris Benson for secretarial support. This research was supported by a grant from Eli Lilly and Company and by MH41115 (AJR).

We also thank Howard P. Roffwarg, M.D., for assistance in designing the study and in obtaining funding for the project.

\section{REFERENCES}

Armitage R, Rush AJ, Trivedi M, Cain J, Roffwarg HP (1994): The effects of nefazodone on sleep architecture in depression. Neuropsychopharmacology 10:123-127

Baloh RW, Dietz J, Spooner JW (1982): Myoclonus and ocular oscillations induced by L-tryptophan. Ann Neurol 11:95-97

Cooper GL (1988): The safety of fluoxetine-an update. Brit J Psychiatry 153(3):77-86

Depression Guideline Panel (1993): Clinical Practice Guideline Number 5. Depression in Primary Care (vol 2). Treatment of Major Depression, AHCPR Publication No. 930551, Rockville, MD: U.S. Dept. of Health and Human Services, Public Health Service, Agency for Health Care Policy and Research.

Dorsey CM, Cunningham SL, Lukas SE, Winkelman JW, Richardson GS, Jones K (1992): Fluoxetine-induced eye movements during non-REM sleep. Sleep Res 21:55

Ellison JM, Stanziani P (1993): SSRI-associated nocturnal bruxism in four patients. J Clin Psychiatry 54:432-434

Fishbain DA, Dominquez M, Goldberg M, Olsen E, Rosomoff $H$ (1992): Dyskinesia associated with fluoxetine use: Case report. Neuropsychiatr, Neuropsychol, Behav Neurol 5:97-100

Hamilton MA (1960): A rating scale for depression. J Neurol Neurosurg Psychiatry 12:56-62

Hendrickse WA, Roffwarg HP, Grannemann B, Orsulak PJ, Armitage R, Cain JW, Battaglia J, Debus JR, Rush A] (1994): The effects of fluoxetine on the polysomnogram of depressed outpatients: A pilot study. Neuropsychopharmacology 10:85-91

Hoffmann RF, Moffitt AR, Shearer JC, Sussman PS, Wells RB (1979): Conceptual and methodological considerations towards the development of computer-controlled research on the electrophysiology of sleep. Waking and Sleeping 3:1-16

Keck, PE Jr., Hudson JI, Dorsey CM, Campbell PI (1991): Effect of fluoxetine on sleep. Biol Psychiatry 29:618 
Keck, PE Jr., Hudson JI, Dorsey CM, Matheson JK, Campbell PI (1990): Increased eye movements during non-REM sleep in fluoxetine-induced insomnia. Sleep Res 20:66

Lipinski JF, Mallya G, Zimmerman P, Pope HG (1989): Fluoxetine-induced akathisia: Clinical and theoretical implications. J Clin Psychiatry 50:339-342

Nicholson AN, Pascoe PA (1988): Studies on the modulation of sleep-wakefulness continuum in man by fluoxetine, a 5-HT uptake inhibitor. Neuropharmacology 27:597-602

Rechtschaffen A, Kales A (eds) (1968): A Manual of Standardized Terminology, Techniques and Scoring System for Sleep Stages of Human Subjects, National Institute of Health Publication 204, Washington, DC, U.S. Government Printing Office

Schenck CH, Mahowald MW, Kim SW, O'Connor KA, Hur- witz TD (1992): Prominent eye movements during NREM sleep and REM sleep behavior disorder associated with fluoxetine treatment of depression and obsessive-compulsive disorder. Sleep 15:226-235

Seibyl JP, Glazer WM, Innis RB (1989): Serotonin function in tardive dyskinesia. Psychiatr Ann 19:310-314

Spitzer RL, Williams JBW, Gibbons M (1986): The Structured Clinical Interview for DSM-III-R (SCID), New York: New York Psychiatric Institute

Tychsen L, Sitaram N (1989): Catecholamine depletion produces irrepressible saccadic eye movements in normal humans. Ann Neurol 25:444-449

Winkelman J, Dorsey C, Cunningham S, Lukas S, Richardson $G$ (1992): Fluoxetine produces persistent rapid eye movements in non-REM sleep. Sleep Res 21:78 